\title{
Temporomandibular joint involvement in children with juvenile idiopathic arthritis
}

\author{
A single tertiary-center experience
}

Jubran T. Alqanatish, MD, MME, Banan S. Alrewaithi, MD, Wafaa M. Alsewairi, MD, Altaf H. Khan, MS, Mohammed J. Alsalman, MD, Abdulrhman A. Alrasheed, MD.

\begin{abstract}

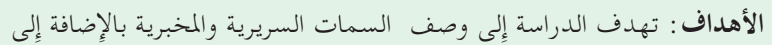

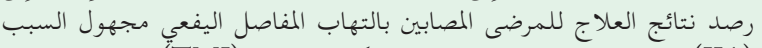

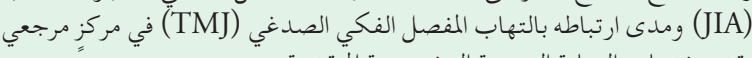

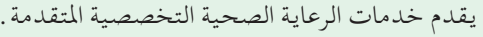

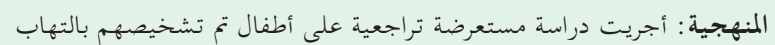

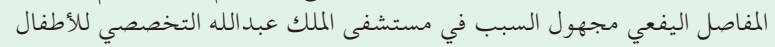

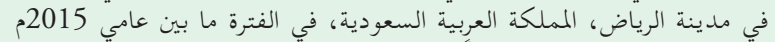

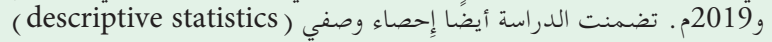

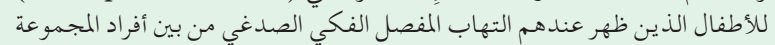

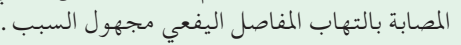

النتائج: تم استعراض 123 حالة مصابة بالتهاب المفاصل اليفعي مجهول

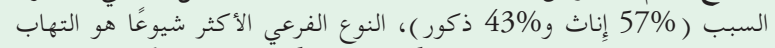

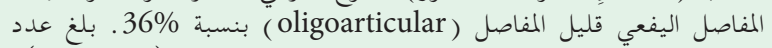

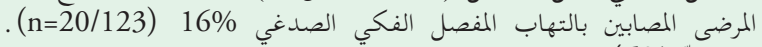

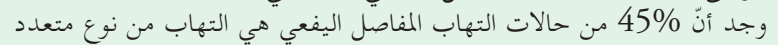

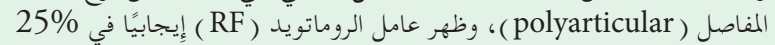

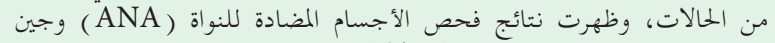

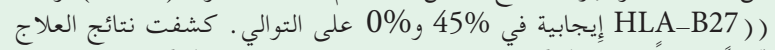

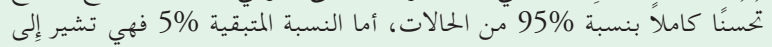
حدوث مضاعفات . ماندة

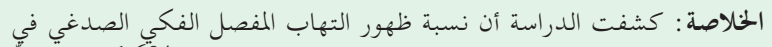

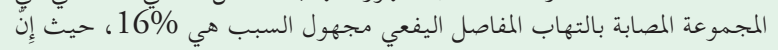

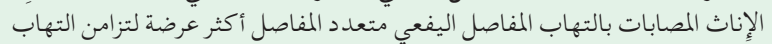

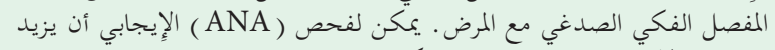

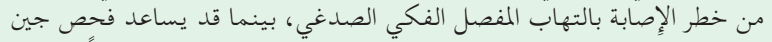
(HLA-B27)

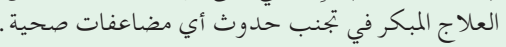

Objectives: To describe the clinical and laboratory characteristic, state the treatment and outcome of patients with juvenile idiopathic arthritis (JIA), and describe temporomandibular joint (TMJ) involvement as observed in a large tertiary center.

Methods: A retrospective cross-sectional study of children diagnosed with JIA was assessed at King Abdullah
Specialist Children's Hospital, Riyadh, Saudi Arabia (2015-2019), which included a descriptive analysis of children who had TMJ involvement among our study group. Subjects diagnosed with the TMJ arthritis were based either on clinical musculoskeletal examination or using contrast-enhanced MRI.

Results: We reviewed 123 cases with different JIA subtypes (57\% females). The most frequent subtype is the oligoarticular (36\%). TMJ involvement was found in $16 \%(n=20 / 123)$ of the patients, of whom $45 \%$ had Polyarticular JIA. The rheumatoid factor was positive in 25\%; antinuclear antibody (ANA) in $45 \%$ and none showed positivity to HLAB27. Treatment resulted in complete resolution in 95\% of cases, while Micrognathia and obstructive sleep apnea were the complications reported in $5 \%$ of cases.

Conclusion: TMJ involvement in JIA is not uncommon. Females with polyarticular disease were more frequently affected with TMJ arthritis. Positive ANA could be a risk factor for TMJ involvement, while positive HLAB27 might have some protective effects. Early treatment for TMJ arthritis is essential to avoid possible complications.

Keywords: temporomandibular joint, juvenile idiopathic arthritis, children, Saudi Arabia

Saudi Med J 2021; Vol. 42 (4): 399-404

doi: 10.15537/smj.2021.42.4.20200470

From King Saud bin Abdulaziz University for Health Sciences (Alqanatish, Alrewaithi, Alsewairi, Alsalman, Alrasheed); from King Abdullah International Medical Research Center (Alqanatish, Alsewairi, Khan, Alsalman, Alrasheed); from King Abdullah Specialist Children's Hospital (Alqanatish, Alsewairi, Alrasheed); and from King Abdulaziz Medical City (Alsalman), Riyadh, Kingdom of Saudi Arabia.

Received 17th November 2020. Accepted 10th March 2021.

Address correspondence and reprint request to: Dr. Jubran Alqanatish, Department of Pediatrics, King Abdullah Specialist Children's Hospital, Riyadh, Kingdom of Saudi Arabia. E-mail: qanatishj@ngha.med.sa ORCID ID: http:/lorcid.org/0000-0001-6467-6972 
$\mathcal{T}$ uvenile idiopathic arthritis (JIA) is the most common chronic inflammatory arthritis in children. Juvenile idiopathic arthritis is a term used to describe a heterogeneous group of autoimmune diseases leading to synovial-related inflammations of unknown etiology, which begins before the age of 16, affects at least one joint and persists for not less than 6 weeks. ${ }^{1-3}$ Juvenile idiopathic arthritis could affect any of the axial or peripheral body joints and is classified according to the International League of Associations for Rheumatology (ILAR) into 7 subtypes based on the pattern of arthritis development during the initial 6-month interval, which include systemic-onset, oligoarticular, rheumatoid factor (RF)-positive polyarthritis, RF-negative polyarthritis, enthesitis-related, psoriatic, and undifferentiated arthritis. ${ }^{2}$ The diagnosis of JIA is a diagnosis of exclusion, which mainly depends on a clinical assessment with little to no role of laboratory tests or supplementary imaging studies. However, the laboratory and imaging studies would be helpful in subtype classification. ${ }^{3}$

Children diagnosed with JIA often develop inflammation of the temporomandibular joint (TMJ). Temporomandibular joint is a joint that connects the skull with the lower jaw. An articular disc made up of fibrous connective tissue divides the joint space into upper and lower compartments. The movements that take place in this joint is quite complex, rotary or hinge movement around a horizontal axis during mouth opening through the center of condylar heads bilaterally. And gliding or translatory movement of the mandible in the anteroposterior and/or mediolateral direction. Gliding or translator movement occur at the upper compartment while the lower compartment allows rotary or hinge movement. ${ }^{4}$ The prevalence of TMJ arthritis in children with JIA was reported to be up to $87 \% .^{5-11}$

Untreated TMJ arthritis in children can lead to mandibular growth limitation, which causes jaw asymmetry, malocclusion, and limited maximal incisal opening (MIO). ${ }^{11-13}$ Diagnosis of TMJ involvement in children with JIA remains difficult, thereby it would be beneficial to be able to identify children who are at high risk for TMJ involvement. ${ }^{10}$ Providers could refer and intervene earlier and would likely help prevent

Disclosure. Authors have no conflict of interests, and the work was not supported or funded by any drug company. progression. Temporomandibular joint involvement may be present in all JIA subtypes and, occasionally, it is the only joint involved. ${ }^{11-14}$ Among local studies, clinical and laboratory manifestations of a total of 115 patients diagnosed with JIA for over a period of 15 years revealed that systemic and polyarticular subtypes were the most common. Temporomandibular joint involvement was not determined in this study. ${ }^{15}$

Another retrospective study of 82 patients diagnosed with JIA between 2007 and 2015 showed that systemic onset and polyarticular type were the most common types, respectively. However, TMJ involvement was also not considered in this study. ${ }^{16}$

In a US study among 330 JIA patients, only 6 patients had evidence of synovitis and/or degenerative changes of TMJ and were diagnosed with TMJ arthritis, and it was found to be most common in female, Caucasians, HLA-B27 negative, antinuclear antibody (ANA) negative, poly RF-negative subtype, with multiple joint involvement. ${ }^{11}$

The TMJ is the least diagnosed joint in children with JIA and their arthritis could result in permanent functional and cosmetic complications. Since the frequency of TMJ involvement among the different ILAR categories of JIA patients in the local study data is yet to be determined, we decided to conduct a retrospective review of all patients diagnosed with JIA at our institute to observe the frequency of TMJ involvement, define the most common type of JIA associated with the condition, as well as to identify possible risk factors and describe the treatment and outcome of TMJ arthritis.

Methods. The study design is a retrospective study. The study is a descriptive analysis to find the percentage of TMJ involvement in children diagnosed with JIA.

The study was carried out in the Pediatric Rheumatology Clinic, King Abdullah Specialist Children's Hospital (KASCH), Riyadh, Saudi Arabia. We included all the subjects who were diagnosed with JIA from 2015 to 2019.

We utilized the ILAR criteria to include the subjects with JIA, whereas subjects diagnosed with the TMJ arthritis were based either on clinical musculoskeletal examination or using contrast-enhanced MRI. All patients should have no less than 2 clinic visits.

The demographics and clinical variables in this study were: age, gender, age at onset of JIA, type of JIA, age of TMJ involvement, laterality of TMJ involvement, clinical presentation (pain, tenderness, deviation of the mandible, decreased mouth opening), ANA and RF, presence of HLA-B27, diagnostic magnetic resonance 
imaging (MRI), systemic treatment (non-steroidal antiinflammatory drugs (NSAIDs), systemic corticosteroids, disease-modifying anti-rheumatic drugs (DMARDs), biological therapy) and local intra-articular steroid injection used during the course of the disease and the outcome (improved, or developed complication). On the one hand, the patient's improvement was considered if there were no more symptoms and signs or mild signs and symptoms of arthritis in the TMJ that did not interfere with function. On the other hand, TMJ complication was considered if there were residual sequelae that affect the function or appearance of TMJ and make the patient a candidate for maxillofacial referral intended for possible intervention.

The study is about the children diagnosed with JIA and TMJ. For the inclusion of subjects with JIA disease, we applied the ILAR criteria. The diagnosis for the subjects with TMJ involvement were using clinical assessment and/or radiological modalities, the sources of biases may be due to the experiences of the radiologist as well as the clinician working experiences. To our understanding there may be some minimal biases.

We included all the subjects diagnosed with JIA and TMJ diseases during the period of 2015 to 2019, as well as the study is solely descriptive. Hence, there was no need to rely on sample size estimation.

Statistical analysis. The continuous variable were described as mean \pm SD (standard deviation), and the categorical variables as count with percentage (\%). The

Table 1 - Patient disease characteristics in total study group and temporomandibular joint (TMJ) group.

\begin{tabular}{lcc}
\hline Patient's characteristics & $\begin{array}{c}\text { Total study } \\
\text { group } \\
(\mathbf{n}=123)\end{array}$ & $\begin{array}{c}\text { TMJ group } \\
(\mathbf{n}=20)\end{array}$ \\
\hline Gender & 57 & 70 \\
Female & 43 & 30 \\
Male & \\
Type of Juvenile idiopathic arthritis & (JIA) (\%) & \\
Oligoarticular & 36 & 20 \\
Poly JIA & 22 & 45 \\
SOJIA & 20 & 15 \\
ERA & 14 & 15 \\
Psoriatic arthritis & 6 & 5 \\
Undifferentiated arthritis & 3 & None \\
Blood tests & & \\
Antinuclear antibody & 38 & 45 \\
Rheumatoid factor & 5 & 25 \\
HLAB27 & 5 & None \\
Use of biological treatment & 73 & 100 \\
\hline
\end{tabular}

SOJIA: systemic-onset juvenile idiopathic arthritis, ERA: enthesitis-related arthritis statistical analysis was carried out using SAS version 9.4 SAS Institute, North Carolina, USA (a statistical software package).

The study was approved by the Institutional and Ethics Review Board of King Abdullah International Medical Research Center (KAIMRC).

Results. A total of 123 children were diagnosed with JIA. The distribution by gender was 53 (43\%) boys and $70(57 \%)$ girls. The most frequent JIA subtype was oligoarticular (36\%), while the other types were poly JIA (22\%), Systemic-onset juvenile idiopathic arthritis (SOJIA) (20\%), enthesitis-related arthritis (ERA) $(14 \%)$, psoriatic arthritis (6\%), and undifferentiated arthritis (3\%).

The mean age for the diagnosis of JIA was $13.5 \pm$ 3.63 years old, and the mean age of TMJ involvement was $11 \pm 4.00$ years old, which are $40 \%$ younger than 10 years old and $60 \%$ older than 10 years old. The number of patients with TMJ involvement was $20(16 \%)($ females $=14[70 \%]$, males $=6[30 \%])$. Of these patients with TMJ, 45\% had polyarticular JIA, including $20 \%$ poly JIA RF-positive and $25 \%$ poly JIA RF-negative. Other subtypes showed oligoarticular JIA $20 \%$, SOJIA $15 \%$, ERA $15 \%$, and psoriatic arthritis $5 \%$ (Table 1).

The diagnosis of TMJ arthritis was made after clinical presentation with pain on TMJ in $80 \%$ of cases. Other symptoms included having a click in $15 \%$ and tenderness in $25 \%$ of cases. Deviation of the mandible was reported in $50 \%$ of cases. Bilateral TMJ arthritis was reported in half of the patients and in the other $50 \%$ (30\% unilateral on the right side and $20 \%$ on the left side).

Eleven patients had contrast-enhanced MRI, 7 of them had signs of inflammation, and 4 were unremarkable. Nine children had clinical signs of TMJ pathology on physical examination, but an MRI had not been performed for various reasons, with family refusal of general anesthesia being the most frequent cause. Antinuclear antibody test was positive in 45\% and none showed positivity for HLAB27. Rheumatoid factor was positive in $25 \%$ (Table 1 ).

Systemic medication used in patients with TMJ involvement at any time during the course of the disease included NSAIDs administered to $50 \%$ of patients, systemic corticosteroids in 55\%, disease-modifying anti-rheumatic drugs (DMARDs) in 95\% including methotrexate (MTX) in 85\%, hydroxychloroquine in $20 \%$, sulfasalazine in $10 \%$, and mycophenolate mofetil (MMF) in 10\%. Almost $100 \%$ of the patients with TMJ involvement had biological medications 
used as tumor necrosis factor- $\alpha$ (TNF- $\alpha$ ) antagonists, such as infliximab administered to $25 \%$ of patients, adalimumab used in $40 \%$ of cases. Similarly, $25 \%$ of the patients received abatacept and the same percentage received tocilizumab, while $5 \%$ of the patients received secukinumab and the same percentage received ustekinumab (Table 1).

Local intra-articular corticosteroid injections were carried out in only $25 \%$ of the patients, and all of them were confirmed to have TMJ arthritis in the MRI.

In our study, treatment resulted in complete improvement in $95 \%$ of cases, while micrognathia and obstructive sleep apnea were the complications reported in $5 \%$ of cases (Figure 1).

Discussion. The local data from Saudi Arabia on TMJ involvement among children affected with JIA is lacking. The frequency of TMJ arthritis and risk factors for this disease were not described in the 2 previously published cohorts from Saudi Arabia. ${ }^{15,16}$

In previous studies, estimates of TMJ involvement in JIA have ranged from $17 \%$ to $87 \%$ due to variation in assessment and examination methods. ${ }^{5-11}$ In our study across all types of JIA, 16\% of patients have TMJ involvement ( $70 \%$ are females). The most common type of JIA with TMJ involvement was found to be polyarticular JIA, RF-negative in $25 \%$ of cases and the least common type was psoriatic arthritis in only $5 \%$ of cases. The rate of TMJ involvement in our cohort was lower $(16 \%)$ than the rates reported in previous studies. In addition, our study indicates a 15\% TMJ involvement being reported in SOJIA; a higher and lower rates was previously reported. ${ }^{8,17-21}$ We found $50 \%$ of the patients having a bilateral disease that is mostly in a polyarticular type.

In a previous study by Cannizzaro et al, ${ }^{18}$ among 223 JIA patients, 86 (39\%) patients had developed TMJ arthritis (75\% were female) and the most commonly affected type was extended oligoarticular JIA in $61 \%$, while the least affected type was enthesitis-related arthritis in $11 \%$. In contrast, our cohort oligoarticular JIA arthritis is only $20 \%$ and enthesitis-related in $15 \%$.

In another study, Abramowicz et $\mathrm{al}^{11}$ reported a lower frequency of TMJ involvement among JIA categories in only $10 \%$ of 60 symptomatic patients patients, and the majority of them were females (87\%). Polyarticular RF-negative was reported to have a higher TMJ involvement in $32 \%$ and the least subtypes were psoriatic $(3 \%)$ and undifferentiated $(3 \%)$. These findings coincide with those of our cohort.

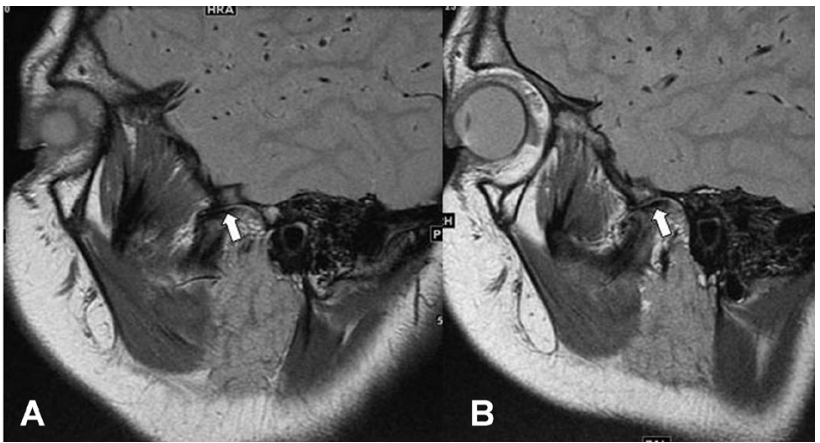

Figure 1 - Sagittal MR proton density-weighted image showing A) right temporomandibular joint (TMJ) (closed mouth). There is irregularity with flattening of the articular surface of the mandibular condyle with volume loss of the disc (white arrow). B) left TMJ (closed mouth). There is irregularity with flattening of the articular surface of the mandibular condyle with volume loss of the disc (white arrow)

Temporomandibular joint arthritis was discovered to be presented with pain in $80 \%$ of the cases, clicking of the jaw in $15 \%$, and asymptomatic in $5 \%$ of cases. The physical examination findings were tenderness of TMJ in $25 \%$, deviation of the mandible in $50 \%$, and $50 \%$ of the cases have decreased mouth opening.

Clinical examination or self-report only provides poor performance of TMJ arthritis. ${ }^{22}$ Therefore, the possibility of detecting real TMJ arthritis is improved by combining physical examination and the use of images. ${ }^{23}$ Some studies have shown a high prevalence of TMJ arthritis using MRI in early-onset JIA even among asymptomatic children. ${ }^{24,25}$ Magnetic resonance imaging in our cohort showed TMJ synovitis in 7 patients. However, MRI was only performed in 55\% of patients with clinically suspected TMJ arthritis due to different factors related mainly to the family's unwillingness to subject the child to general anesthesia.

In Cannizzaro et $\mathrm{al}^{18}$ cohort, HLA-B27 was positive in $3 \%$ of patients with TMJ arthritis and was also positive in $18 \%$ in the study of Abramowicz et al. ${ }^{23}$ These 2 subsequent findings are inconsistent with the findings in our cohort because the low prevalence of HLA-B27 in the general population of Saudi Arabia was assessed using cord blood and healthy organ transplant donor databases. ${ }^{11,18-26}$

Systemic medications used during the course of this disease included NSAIDs administered to $50 \%$ of patients, systemic corticosteroids in 55\%, DMARD in 95\% including methotrexate (MTX) in 85\%, hydroxychloroquine in $20 \%$, sulfasalazine in $10 \%$ and mycophenolate mofetil (MMF) in 10\%. Almost 100\% 
of the patients with TMJ involvement had biological medications used as tumor necrosis factor- $\alpha$ (TNF- $\alpha$ ) antagonists such as infliximab administered to $25 \%$ of the patients and adalimumab used in $40 \%$ of the cases. Similarly, $25 \%$ of the patients received abatacept and another $25 \%$ of the patients also received tocilizumab, while $5 \%$ of the patients received secukinumab and the same percentage of patients received ustekinumab.

It seems that in our cohort, TMJ arthritis is more frequent in JIA that has a severe course because almost all patients have been treated with biologic therapy as mentioned above. This could be different from the findings of other researchers, as Cannizzaro et $\mathrm{al}^{18}$ found that only $40 \%$ required tumor necrosis factor- $\alpha$ (TNF- $\alpha$ ) antagonists, while Abramowicz et $\mathrm{al}^{23}$ reported the use of biological products (adalimumab, etanercept, and infliximab) in only $24 \% .{ }^{11}$

In Saudi children that we described, they are older when they are diagnosed with JIA (mean age at onset of JIA was 13.5 years); therefore, they experience less growth disturbances. They also receive more aggressive treatment including biologics which may actually prevent TMJ damage. Only $5 \%$ of our patients sustained chronic sequelae of TMJ arthritis. The current study is limited due to its inherent retrospective nature; MRI was performed in only about half of our patients with clinically suspected TMJ arthritis. It is possible if MRI was performed in all subjects in our JIA cohort including asymptomatic patients additional subjects would have been identified. Given the risks of general anesthesia, some of the parents had understandably refused the MRI for their children. It is a really important point for rheumatologist to provide a sufficient information to children and their parents for performing MRI under general anesthesia as this has been found to be effective in relieving child and parents anxiety. Not to mention, the mouth opening assessment was not performed objectively in most patients.

In conclusion, TMJ involvement occurs in all JIA subtypes. The disease is more bilateral in our cohort and mostly in the polyarticular type. Females with polyarticular RF-negative subtype and HLA-B27 negative are more at risk of TMJ arthritis in our cohort. Therefore, careful assessment of TMJ arthritis and early treatment are required in this vulnerable group.

\section{References}

1. Petty RE, Southwood TR, Baum J, Bhettay E. Revision of the proposed classification criteria for juvenile idiopathic arthritis: Durban. 1997. J Rheumatol 1998; 25: 1991-1994.
2. Petty RE, Southwood TR, Manners P, Baum J, Glass DN, Goldenberg J, et al. International League of Associations for Rheumatology classification of juvenile idiopathic arthritis: second revision, Edmonton, 2001. J Rheumatol 2004; 31: 390-392.

3. Kim KH, Kim DS. Juvenile idiopathic arthritis: Diagnosis and differential diagnosis. Korean J Pediatr 2010; 53: 931.

4. Merlini L, Palla S. The relationship between condylar rotation and anterior translation in healthy and clicking temporomandibular joints. Schweiz Monatsschr Zahnmed 1988; 98: 1191-1199.

5. Ronning O, Valiaho ML, Laaksonen AL. The involvement of the temporomandibular joint in juvenile rheumatoid arthritis. Scand J Rheumatol 1974; 3: 89-96.

6. Pedersen TK, Jensen JJ, Melsen B, Herlin T. Resorption of the temporomandibular condylar bone according to subtypes of juvenile chronic arthritis. J Rheumatol 2001; 28: 2109-2115.

7. Twilt M, Mobers SM, Arends LR, ten Cate R, van SuijlekomSmit L. Temporomandibular involvement in juvenile idiopathic arthritis. J Rheumatol 2004; 31: 1418-1422.

8. Arabshahi B, Cron R. Temporomandibular joint arthritis in juvenile idiopathic arthritis: the forgotten joint. Curr Opin Rheumatol 2006; 18: 490-495.

9. Pedersen TK, Kuseler A, Gelineck J, Herlin T. A prospective study of magnetic resonance and radiographic imaging in relation to symptoms and clinical findings of the temporomandibular joint in children with juvenile idiopathic arthritis. J Rheumatol 2008; 35: 1668-1675.

10. Stoll ML, Sharpe T, Beukelman T, Good J, Young D, Cron RQ. Risk factors for temporomandibular joint arthritis in children with juvenile idiopathic arthritis. J Rheumatol 2012; 39: 1880-1887.

11. Abramowicz Sh, Levy JM, Prahalad S, Travers C, Angeles-Han ST. Temporomandibular joint involvement in children with juvenile idiopathic arthritis: a preliminary report. Oral Surg Oral Med Oral Pathol Oral Radiol 2019; 127: 19-23.

12. Ronchezel MV, Hilário MO, Goldenberg J, Lederman HM, Faltin K Jr, de AzevedoMF, et al. Temporomandibular joint and mandibular growth alterations in patients with juvenile rheumatoid arthritis. J Rheumatol 1995; 22: 1956-1961.

13. Ringold S, Cron RQ. The temporomandibular joint in juvenile idiopathic arthritis: Frequently used and frequently arthritic. Pediatr Rheumatol Online J 2009; 7: 11.

14. Martini G, Bacciliero U, Tregnaghi A, Montesco MC, Zulian F. Isolated temporomandibular synovitis as unique presentation of juvenile idiopathic arthritis. J Rheumatol 2001; 28: 1689-1692.

15. Bahabri S, Al-Sewairi W, Al-Mazyad A, Karrar A, Al-Ballaa S, El-Ramahai K, et al. Juvenile rheumatoid arthritis: The Saudi Experience. Ann Saudi Med 1997; 17: 413-418.

16. Al-Hemairi M, Albokhari S, Muzaffer M. The Pattern of Juvenile Idiopathic arthritis in a single tertiary center in Saudi Arabia. Int J Inflamm 2016; 2016: 1-8.

17. Billiau AD, Hu Y, Verdonck A, Carels C, Wouters C. Temporomandibular joint arthritis in juvenile idiopathic arthritis: prevalence, clinical and radiological signs, and relation to dentofacial morphology. J Rheumatol 2007; 34: 1925-1933.

18. Cannizzaro E, Schroeder S, Muller LM, Kellenberger CJ, Saurenmann RK. Temporomandibular joint involvement in children with juvenile idiopathic arthritis. J Rheumatol 2011; 38: 510-515. 
19. Kuseler A, Pedersen TK, Herlin T, Gelineck J. Contrast enhanced magnetic resonance imaging as a method to diagnose early inflammatory changes in the temporomandibular joint in children with juvenile chronic arthritis. J Rheumatol 1998; 25: 1406-1412.

20. Kuseler A, Pedersen TK, Gelineck J, Herlin T. A 2-year follow-up study of enhanced magnetic resonance imaging and clinical examination of the temporomandibular joint in children with juvenile idiopathic arthritis. J Rheumatol 2005; 32: 162-169.

21. Resnick C, Pedersen TK, Abramowicz S, Twilt M, Stoustrup $\mathrm{PB}$. Time to reconsider management of the temporomandibular joint in juvenile idiopathic arthritis. J Oral Maxillofac Surg 2018; 76: 1145-1146.

22. Kristensen KD, Stroustrup P, Kuseler A, Pedersen TK, Twilt M, Herlin T. Clinical predictors of temporomandibular joint arthritis in juvenile idiopathic arthritis: a systematic literature review. Semin Arthritis Rheum 2016; 45: 717-732.
23. Abramowicz S, Kim S, Prahalad S, Chouinard AF, Kaban LB. Juvenile arthritis: current concepts in terminology, etiopathogenesis, diagnosis, and treatment. Int J Oral Maxillofac Surg 2016; 45: 1723-1727.

24. Weiss PF, Arabshahi B, Johnson A, Bilaniuk LT, Zarnow D, Cahill AM, et al. High prevalence of temporomandibular joint arthritis at disease onset in children with juvenile idiopathic arthritis, as detected by magnetic resonance imaging but not by ultrasound. Arthritis Rheum 2008; 58: 1189-1196.

25. Stoll ML, Kau CH, Waite PD, Cron RQ. Temporomandibular joint arthritis in juvenile idiopathic arthritis, now what? Pediatr Rheumatol 2018; 16: 32.

26. Omair MA, AlDuraibi FK, Bedaiwi MK, Abdulaziz S, Husain W, El Dessougi M, et al. Prevalence of HLA-B27 in the general population and in patients with axial spondyloarthritis in Saudi Arabia. Clin Rheumatol 2017; 36: 1537-1543.

\section{Withdrawal policy}

By submission, the author grants the journal right of first publication. Therefore, the journal discourages unethical withdrawal of manuscript from the publication process after peer review. The corresponding author should send a formal request signed by all co-authors stating the reason for withdrawing the manuscript. Withdrawal of manuscript is only considered valid when the editor accepts, or approves the reason to withdraw the manuscript from publication. Subsequently, the author must receive a confirmation from the editorial office. Only at that stage, authors are free to submit the manuscript elsewhere.

No response from the authors to all journal communication after review and acceptance is also considered unethical withdrawal. Withdrawn manuscripts noted to have already been submitted or published in another journal will be subjected to sanctions in accordance with the journal policy. The journal will take disciplinary measures for unacceptable withdrawal of manuscripts. An embargo of 5 years will be enforced for the author and their co-authors, and their institute will be notified of this action. 